\title{
Demonstration of Label-Rewriting using 1 x 4 All-Optical Packet Switch employing Optical Processing of Scalable In-Band Labels
}

\author{
H.D. Jung, N. Calabretta, J. Herrera, E. Tangdiongga, A.M.J. Koonen and H.J.S. Dorren \\ COBRA Research Institute, Eindhoven University of Technology, P.O. Box 513, 5600 MB - Eindhoven, NL \\ jhd94@ieee.org
}

\begin{abstract}
We demonstrate label-rewriting function using $1 \times 4$ all-optical packet-switch that utilizes a highly scalable and asynchronous label processor. Results show that labels are successfully exchanged and the process causes only $0.5 \mathrm{~dB}$ power-penalty and error-free operation at $160 \mathrm{~Gb} / \mathrm{s}$.
\end{abstract}

Keywords: Optical packet switching, Optical processing

\section{Introduction}

All-optical packet switching is expected to be a promising candidate to solve the mismatch between the fiber bandwidth and router forwarding capacity at bit-rate where electronics is too slow to directly process the data [1]. In an all-optical packet switch, the packets are routed based on address information that is encoded by attached labels.

A key issue in the implementation of all-optical packet switch is to reduce the total number of optical switches required for the realization of the packet switched cross-connect. For instance, if $1 \times 2$ optical switch is used, the total of optical switches is proportional to $N^{2}$ because the number of optical switches needed at each fiber input in a NxNWDM switching node is $N^{2}$, where $N$ is the number of the input port. However if $1 \times N$ optical switch is available, the total number of optical switches required for the cross-connect would be proportional to $N$ and thus drastically decreased. To implement $1 x N$ optical packet switch, it is essential to realize a highly scalable label processing technique that can process $N$ labels in parallel.

Recently, we reported a $1 \times 4$ optical packet switch based on a new scalable label processor for encoded in-band labelling addresses [2]. In [2], we have demonstrated all-optical label erasing and processing. To accomplish the label swapping technique, however, a label-rewriting function that provides a new label from the old one needs to be realized.

In this paper, we demonstrate a label-rewriting function capable of providing a new label based on the old input label. The scheme can be built using a similar set-up employed for the label processor demonstrated in [2]. The performance of the label-rewriting function was evaluated by the power penalty at each node of two cascaded nodes.

\section{System Operation}

Figure 1 shows the experiment setup for label-rewriting. Packet payload is generated by time-multiplexing a $40 \mathrm{~Gb} / \mathrm{s}$ return-to-zero data-stream carrying $2^{7}-1$ PRBS data at $\lambda_{p}=1548.9 \mathrm{~nm}$ up to $160 \mathrm{~Gb} / \mathrm{s}$ data-stream using a passive fiber-based pulse interleaver. Each bit has duration of $1.6 \mathrm{ps}$ making the $20 \mathrm{~dB}$ bandwidth of the payload to be $5 \mathrm{~nm}$. The resulting packet payload consists of a $250 \mathrm{~ns}$ data burst. The

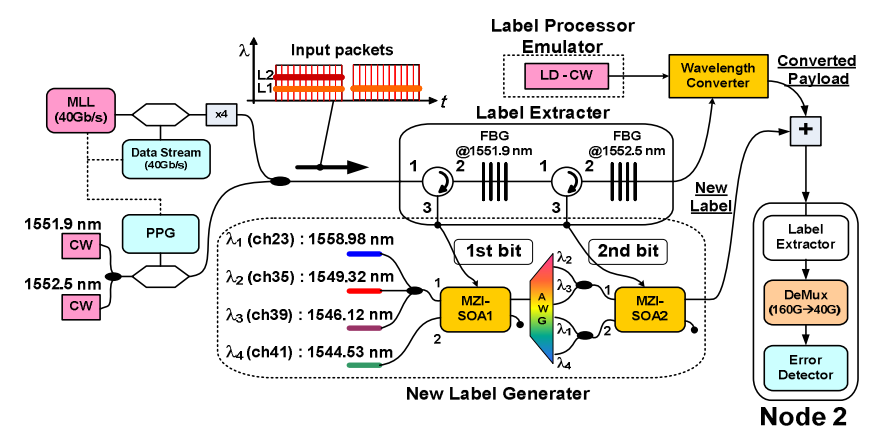

Fig. 1 Experiment setup

packet-to-packet guard time is $10 \mathrm{~ns}$, making the packet repetition rate $260 \mathrm{~ns}$.

We encode addresses by using two labels with different in-band wavelength, which enables to encode 4 different addresses. The in-band label has the advantage that the labels can be extracted by passive wavelength filtering, which simplifying the label processing. Moreover, using a label that has the same time-duration as the packet-payload makes the use of an optical flip-flop redundant. In the experiment, we used two labels with the wavelengths of $\lambda_{L 1}=1551.9 \mathrm{~nm}$ and $\lambda_{L 2}=1552.5 \mathrm{~nm}$ which are within the 20-dB optical bandwidth of the packet-payload.

The label re-writer consists of an all-optical label extractor (used in common with the label processor), an all-optical new label generator that has the same structure as the label processor in [2] and a passive optical coupler. The input packets to the packet switch are firstly processed by the label extractor. The label extractor consists of two fiber (reflective) Bragg gratings (FBG) with a $3 \mathrm{~dB}$ bandwidth of $0.12 \mathrm{~nm}$ and $0.432 \mathrm{~nm}$ centered at $\lambda_{L 1}$ and $\lambda_{L 2}$ respectively. While the packets pass along the label extractor, the label that fits with the wavelength of each FBG is taken (reflected) out. After the label extraction, the old labels are erased and the payload is fed into the wavelength converter [2].

The new label generator receives the $\mathrm{CW}$-signals as inputs, which represents the new labels. The wavelengths of the $\mathrm{CW}$-signals are set to be in-band with the switched payload (the central wavelength of the payload is set by the label processor[2]). For a given old labels combination, the control wavelength is provided by the label processor, and the new label is provided by the label generator. As an example, if packet with labels ' 01 ' enters the packet switch, the label processor produces a cw-signal at $1560.8 \mathrm{~nm}$ (at which the payload is converted) and the label rewriter produces a new label ' 10 ', which is represented by the cw-signal at $1559 \mathrm{~nm}$, that will be attached to the converted payload. 
Each of the extracted labels is fed into each of the MZI-SOAs of the new label generator. The MZI-SOA acts as a fast wavelength selective switch. As shown in Fig.1, CW signals at $\left(\lambda_{1}, \lambda_{2}, \lambda_{3}\right)$ and $\lambda_{4}$ are applied to the port 1 and 2 of MZI-SOA1, respectively. At output of MZI-SOA1, the signals $\left(\lambda_{1}, \lambda_{2}, \lambda_{3}\right)$ appears. However, if there is the label $\left(\lambda_{L 1}\right)$ at the control input of the MZI-SOA1, the signal $\left(\lambda_{4}\right)$ comes out. The AWG and the $2 \times 1$ couplers are used to separate the pair of CW-signals and feed them to the two input ports of MZI-SOA2. According to the label $\left(\lambda_{L 2}\right)$, the MZI-SOA2 will give a distinct set of new labels at the output. This set of new labels is then combined with the $160 \mathrm{~Gb} / \mathrm{s}$ wavelength converted payload and composed of a new packet.

\section{Experiment and Results}

The experimental setup of the label-rewriting demonstration employing two-bit labels is illustrated in Fig.1. We processed four packets with two-bit labels with pattern of ' 00 ', ' 01 ', ' 10 ', ' 11 ' to cover the all possible combinations. The label bits extracted by the label extractor are shown in Fig.2 (a, b). Figure $3(\mathrm{~b})$ shows the spectrum of the payload signal after label extraction. As compared with Fig. 3(a), the label was erased. Based on two-bit labels, the new label generator gave four sets of new labels shown in Fig.2 (c-f) where ' 0 1 '(old) $\rightarrow$ '1 0 '(new), (c) represented by ch 23; '0 0 ' $\rightarrow$ ' 11 ', $(d, e)$ represented by ch35, ch39; ' 10 ' $\rightarrow$ ' 0 0', no outputs; ' 1 1 ' $\rightarrow$ '0 1', (f) represented by ch 41 . New labels from the label generator were combined with the $160 \mathrm{~Gb} / \mathrm{s}$ wavelength converted payload. The spectrum of the converted payload with new labels is shown in Fig. 3(c). To emulate the output of the label processor in [2], CW signal at $1560.8 \mathrm{~nm}$ was used. The converted payload and new labels are processed in node2. At node2 the quality of the payload is evaluated after the label extractor. (See also the spectrum of Fig.3(d)) Figure 4 shows the BER performance at different position of the switch. The label extractor produces a penalty of less than $0.5 \mathrm{~dB}$. After the wavelength conversion, error-free operation was obtained with $5.5 \sim 7 \mathrm{~dB}$ of penalty compared to the input payload, and $1.5 \mathrm{~dB} \sim 3 \mathrm{~dB}$ of additional penalty as compared with the back-to-back of $160 \mathrm{~Gb} / \mathrm{s}$ wavelength conversion. The extra penalty can be ascribed to the pulse broadening after the label extractor which affects the performance of the wavelength conversion. As shown in figure, the label-rewriting function doesn't cause any critical power penalty.

\section{Conclusion}

In this paper, we demonstrate label-rewriting function using a high scalable and asynchronous label processing structure. In the experiment, the labels are successfully exchanged and the process causes $0.5 \mathrm{~dB}$ of power penalty, achieving error-free operation at $160 \mathrm{~Gb} / \mathrm{s}$. We believe that the new structure is promising as a fundamental building block for future large-scale optical packet switching.

\section{References}

[1] D.J. Blumenthal and et. al., "All-optical label swapping networks and technologies", JLT 18, 2000.

[2] N. Calabretta, and et. al., "1x4 all-optical packet switch at $160 \mathrm{~Gb} / \mathrm{s}$ employing optical processing of scalable in-band address labels", PDP33, OFC2008.

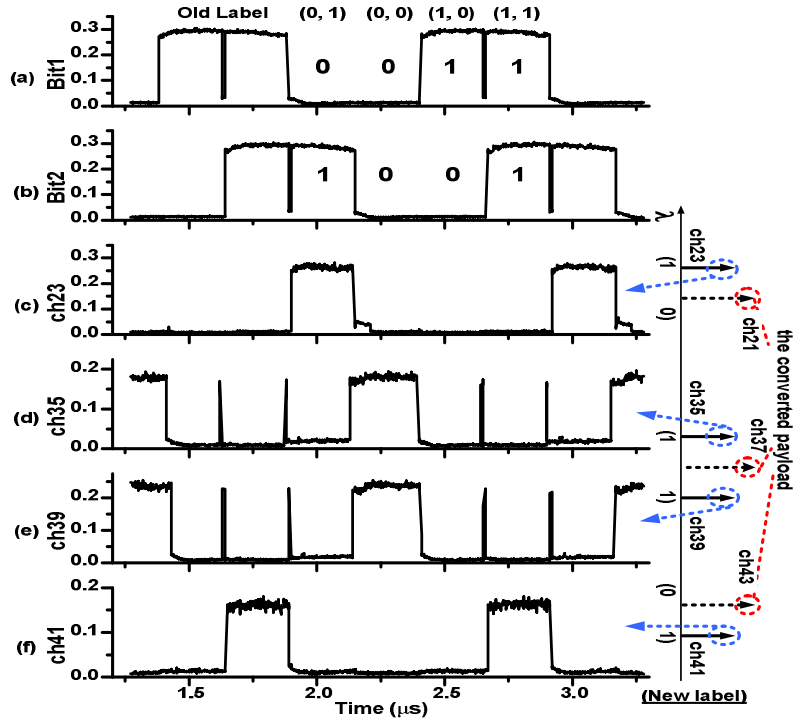

Fig. 2 Traces of outputs from the label extracotr $(a, b)$ and the label-rewriter (c-f).
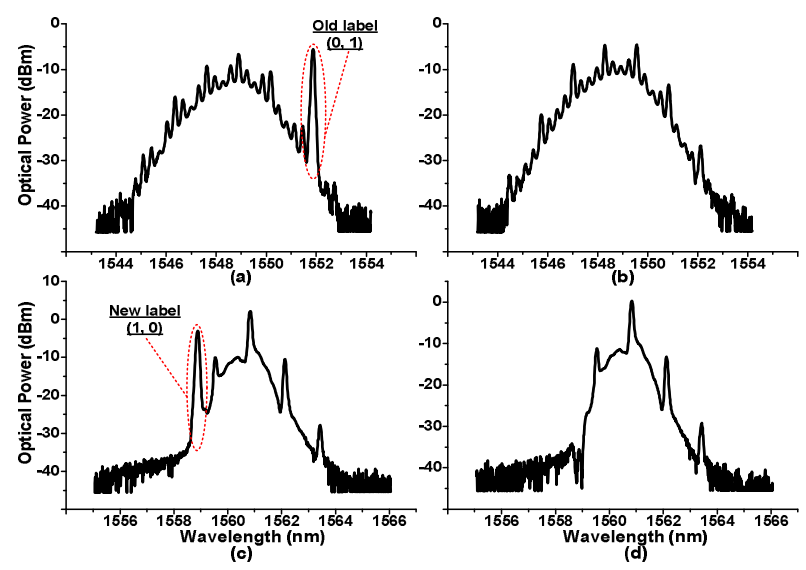

Fig. 3 Optical Spectra (a) Input packet to Node1, (b) Output of label extractor at Node1, (c) Input packet to Node2, (d) Output of label extractor at Node2

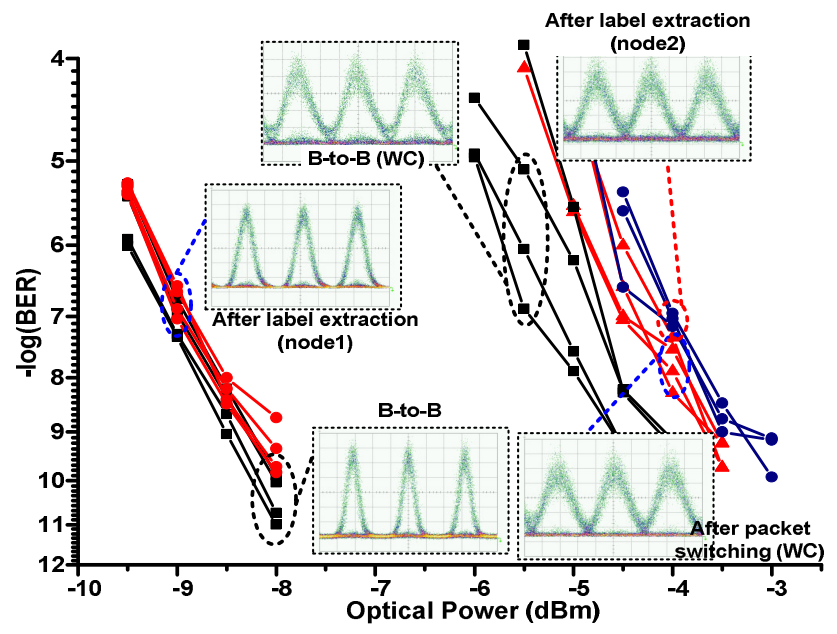

Fig. 4 BER curves and Eye diagrams (Time scale :2ps/div) 